“ (C) 2018 IEEE. Personal use of this material is permitted. Permission from IEEE must be obtained for all other uses, in any current or future media, including

reprinting/republishing this material for advertising or promotional purposes, creating new collective works, for resale or redistribution to servers or lists, or reuse of any copyrighted component of this work in other works." 


\title{
A Solution to the Inverse Pulsed Eddy Current Problem Enabling 3D Profiling
}

\author{
Nalika Ulapane, Linh Nguyen, Jaime Valls Miro and Gamini Dissanayake \\ Centre for Autonomous Systems, Faculty of Engineering and Information Technology \\ University of Technology Sydney, Australia \\ Email: \{nalika.ulapane, vanlinh.nguyen, jaime.vallsmiro, gamini.dissanayake\}@uts.edu.au
}

\begin{abstract}
When a Pulsed Eddy Current (PEC) sensor assesses a metallic surface (i.e., a wall of finite thickness), the inverse problem involves quantification of the geometry and material properties of the wall. Once a PEC sensor is calibrated for a particular material, and the material under test happens to be considerably homogeneous, the inverse problem reduces to quantification of geometry alone. The state-of-the-art in the industry produces a quantification of this geometry only in the form of average wall thickness remaining underneath the sensor footprint, and produces a $2.5 \mathrm{D}$ map containing wall thickness information. Therefore, this paper contributes by proposing a solution that can jointly estimate the remaining wall thickness as well as lift-off (i.e., offset from the sensor to the surface of healthy material), in order to advance PEC sensing outputs by enabling estimation of wall condition in 3D. Since PEC maps are used as inputs for stress calculation and remaining life prediction of certain infrastructure like critical pipes, 3D profiles may become a richer form of input for such applications than 2.5D maps. Since PEC sensing is commonly used to assess ferromagnetic materials, this paper focuses on similar materials as well. The solution is demonstrated in simulation alone and future work should focus on experimental implementations.
\end{abstract}

\section{INTRODUCTION}

Detector coil-based Pulsed Eddy Current (PEC) sensing is widely used for Non-destructive Evaluation (NDE) of metallic structures (typically walls), especially those made of conductive ferromagnetic materials to estimate material thickness remaining under the sensor footprint [1]-[6]. PEC sensing is the most versatile member of the Eddy Current sensing family since its excitation field has a broad frequency spectrum enabling it to tackle the obstacle of skin effect associated with ferromagnetic materials, making the technology usable for NDE of ferromagnetic materials. The technology has been commonly used over the years for NDE of steel in the oil and gas sectors; in recent years there has been an emergence in using it for evaluation of critical water pipes (made of cast irons) as well [7]-[9]. NDE is accomplished by means of solving the inverse PEC problem, which involves estimating the test piece geometry and its electrical and magnetic properties using a PEC signal. Usually, sensors are calibrated for the material being evaluated (some calibration strategies are discussed in [9]), making the problem a lot simpler by minimizing the requirement of estimating electrical and magnetic properties, and reducing to having to estimate

This work was supported by the Critical Pipes Project funded by Sydney Water Cooperation and University of Technology Sydney, Australia. only the geometry. However, the state-of-the-art commercial PEC NDE service providers output estimated geometry only in the form of average wall thickness (i.e., thickness of healthy conductive ferromagnetic material) remaining underneath the sensor footprint, and produces a $2.5 \mathrm{D}$ map containing thickness information. Therefore, this paper contributes by setting the foundation through simulation, to a solution that can jointly estimate the remaining wall thickness as well as lift-off (i.e., offset from the sensor to the surface of healthy material caused by any rust or graphitization), in order to advance PEC sensing outputs by enabling estimation of wall condition in 3D.

The motivation for this work was the fact that PEC sensed outputs being fed as inputs for stress calculation and failure prediction frameworks of certain civil infrastructure like critical water pipes. Therefore, 3D representation of test piece condition may become a richer form of input for such frameworks.

The paper is structured as follows: Section II presents the problem formulation relevant to 3D representation of test piece condition; and Section III proposes a solution to the formulated problem. Since the solution is presented in simulation in this paper, Section IV presents details about the simulation environment used. Section V presents the results obtained to the solution, while concluding remarks and insight towards possible future work are presented in Section VI.

\section{PROBLEM Formulation}

Suppose a PEC sensor is used to assess the remaining wall thickness of a flat conductive ferromagnetic wall in the scenario shown in Fig. 1. Electromagnetic properties of the conductive ferromagnetic material (i.e., magnetic permeability $=\mu$ and electrical conductivity $=\sigma$ ) are known beforehand and the sensor has been calibrated for those properties. No lift-off is present between the wall and the sensor except for the known low lift-off (typically less that $5 \mathrm{~mm}$ ) created by the sensor casing. The nominal thickness $\left(d_{0}\right)$ of the wall is know, and the unknown thickness of healthy conductive ferromagnetic material remaining within the region of interest is $d$, and it remains beneath a layer of corrosion or graphitization whose thickness is $l$ which is also unknown. Provided that $d_{0}>d+l$, there will be another corrosion or graphitization layer whose thickness is $d_{0}-d-l$ beneath the layer of healthy material. In this paper, corrosion or graphitization layers are assumed to be non-magnetic and semi-conductive, which is quite often the 
case in reality. Their relative permeabilities will be such that $\mu_{t} \approx 1$ and $\mu_{b} \approx 1$, making their magnetic permeabilities close to that of air (i.e., $\mu_{0}$ ). However, their electrical conductivities $\sigma_{t}$ and $\sigma_{b}$ will be such that $\sigma_{t}<<\sigma$ and $\sigma_{b}<<\sigma$, but $\sigma_{t}$ and $\sigma_{b}$ falling within the semi-conductive region. Now the problem at hand is: Can $d$ and $l$ be estimated to enable 3D representation of wall condition using a PEC signal calibrated for $\mu$ and $\sigma$, without having to estimate $\mu_{t}, \mu_{b}, \sigma_{t}$ and $\sigma_{b}$ ?

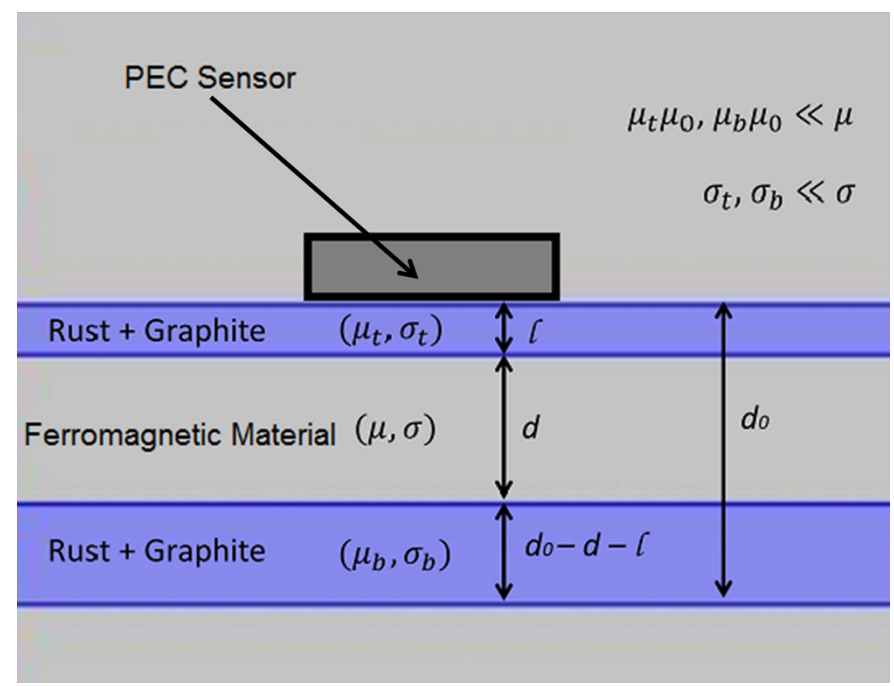

Fig. 1: Scenario for 3D representation of wall condition.

Solving this problem proved to be indeed possible in a simulated environment in the absence of any undesired influences. The solution to the problem and the simulation environment in which the problem is solved are described in the following sections.

\section{Solution to the Problem}

\section{A. PEC Sensor}

Many previous works [2]-[6], [9]-[12] have noted that the detector coil-based PEC sensor architecture is effective in quantifying ferromagnetic material thickness. Since this paper is focused on ferromagnetic materials as well, the detector coil-based architecture is chosen to solve the problem. The sensor simulated in this paper is made up of a concentrically wound, air cored, circular exciter coil and a detector coil, wound using insulated copper wires (Details provided in Section IV). The exciter coil is excited with a current pulse which creates a time varying magnetic field which interacts with the detector coil as well the test piece. The induced voltage in the detector coil is considered as the typical PEC signal which contains information about the test piece geometry and properties. This detector coil voltage takes the shape of an exponential decay and has specific features which can be used for thickness quantification of conductive ferromagnetic materials [4], [9]. Fig. 2 shows the shape of the PEC signals when expressed in logarithmic scale; those signals were generated for the cases $l=2 \mathrm{~mm}$ and $l=14$ $\mathrm{mm}$ (while $\mu_{t}=\mu_{b}=1$ and $\sigma_{t}=\sigma_{b}=0 \mathrm{~S} / \mathrm{m}$ for both $l$ values) in Fig. 1 for grey cast iron (a conductive ferromagnetic material), and were presented in [9]. Variation of signals corresponding to different thickness $(d)$ values can be noticed along with how lift-off influences signals as well, this behaviour depicted in the figure is something common to conductive ferromagnetic materials, not just grey cast iron. This behaviour in the signals is what enables solving the 3D profiling problem for ferromagnetic materials as explained in Subsections III-B and III-C.

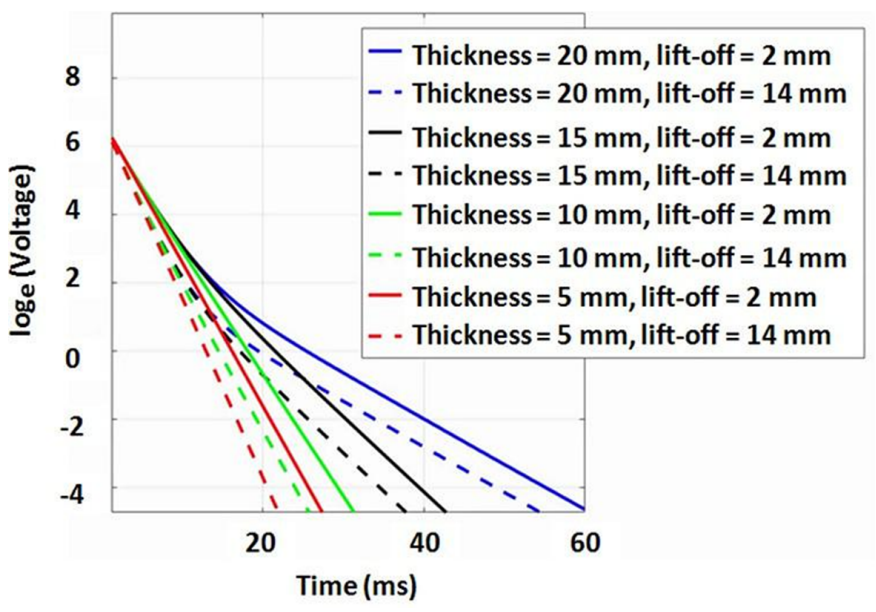

Fig. 2: Simulated PEC Signals.

\section{B. Estimating Thickness (d)}

Suppose a PEC signal is captured on an unknown $d$ and $l$ where $\mu$ and $\sigma$ are known and the sensor has been calibrated for them; then estimating both $d$ and $l$ is possible (as shown later in the paper), without having to estimate $\mu_{t}, \mu_{b}, \sigma_{t}$, and $\sigma_{b}$, under the premise that rust or graphitization layers behave similar to air, provided that $\mu_{t}, \mu_{b} \approx 1$ and $\sigma_{t}, \sigma_{b}<<\sigma$ as specified in Section II. Experiments were done through simulation by setting $\mu_{t}=\mu_{b}=1$ and varying $\sigma_{t}$ and $\sigma_{b}$ from 0 to $0.1 \mathrm{MS} / \mathrm{m}$ to evaluate the validity of this premise. As per the results of these experiments shown in Section V, the rust or graphitization layers proved to have minimal influence on the signal since $\mu$ and $\sigma$ of the conductive ferromagnetic material are much higher than those of the rust or graphitization layers (exact values provided in Section IV). This means rust or graphitization layers effectively behave as air since $\mu_{t}, \mu_{b} \approx 1$ and $\sigma_{t}, \sigma_{b}<<\sigma$, enabling solving the 3D profiling problem with the convenience of not having to estimate electrical and magnetic properties of rust or graphitization layers.

Later stages of PEC signals can be seen to behave as straight lines in Fig. 2. This is again a common behaviour for ferromagnetic materials [9], [11], [12]. It has be shown that the inverse of the absolute value of the gradient of those linear regions (denoted as $\beta$ ) is a function of thickness $d$ and ferromagnetic material properties $\mu$ and $\sigma$. The function takes the form $\beta \approx \mu \sigma d^{2} / \pi^{2}$ [9]. When calibrated for a material (i.e., $\mu$ and $\sigma$ ), $\beta$ can be used as a PEC signal feature to estimate unknown $d$ through a non-linear function in the 
form of $d \approx f(\beta)$, independent of lift-off. Independence of $\beta$ to lift-off is evident in Fig. 2, as the gradient in later stages in all signals hardly vary irrespective of air lift-off changing from $2 \mathrm{~mm}$ to $14 \mathrm{~mm}$. This lift-off independence is still limited by sensor excitation strength though, the lift-off independence holds provided the sensor excitation strength is strong enough for the magnetic fields generated by the sensor to penetrate the test piece despite the lift-off. In this paper, it is considered that the excitation strength (details provided in Section IV) is strong enough to tackle this scenario within the nominal thickness $d_{0}$. Therefore, $d$ can be estimated using $\beta$ independent of lift-off for the purpose of this paper. In this paper, $\beta$ is calculated using the logarithmic signal region between -2 and 0 . Modelling the function $d \approx f(\beta)$ and estimating thickness has been done by non-parametric means in [9] using Gaussian Process (GP) [13]. A similar approach was followed to learn the function through simulated data generated from the simulation model described in Section IV, and to estimate $d$ values provided as results in Section V. The function was learned using simulated data generated by varying $d$ for the $l=0 \mathrm{~mm}$ case, hence the property of $\beta$ being independent of lift-off is exploited to estimate $d$ irrespective of an unknown $l$ being present or not.

\section{Estimating Lift-Off (l)}

The procedure proposed to estimate $l$ can be described as follows:

1. Estimate $d$ as explained in Subsection III-B.

2. Given an estimated $d^{*}$, use the simulation model presented in Section IV to numerically simulate the cases where $l$ takes the value $k\left(d_{0}-d^{*}\right)$, where $k$ takes the values 0 , $0.25,0.5,0.75$ and 1.0 with $\mu_{t}=\mu_{b}=1$ and $\sigma_{t}=\sigma_{b}=0$ $\mathrm{S} / \mathrm{m}$. The intention behind selecting $k$ values is generating five simulated signals corresponding to equally spaced liftoff values between the maximum and minimum viable lift-off values corresponding to the estimated thickness $d^{*}$.

3. For a fixed thickness, note how the signal shifts left when lift-off increases in Fig. 2. Exploit this behaviour to estimate unknown lift-off $l$. Consider a threshold voltage which intersects the later stages of signals (logarithmic voltage $=0$ was chosen for the work of this paper). Extract $t_{t h}$, the threshold crossing time.

4. Learn a non-linear function between $l$ and $t_{t h}$ for the corresponding $d^{*}$ using GP exploiting the five extracted $t_{t h}$ values as training data.

5. Now estimate the unknown lift-off (i.e., $l^{*}$ ) using the learned function between $l$ and $t_{t h}$, using the $t_{t h}$ value extracted from the signal which yielded $d^{*}$, as the testing data point.

Once the above procedure is followed, the unknowns $d^{*}$ and $l^{*}$ will be estimated enabling 3D representation of wall condition, yielding a solution to the problem of interest formulated in Section II. As the possibility of solving the problem is demonstrated through simulation in this paper, Section IV describes the simulation environment used to demonstrate the solution.

\section{Simulation EnVironment}

This section describes the simulation model used in this paper. Shown in Fig. 1 is a pictorial depiction of the simulation model; since the sensor is circular, this was implemented as a 2D-Axisymmetric [11] model in COMSOL Multiphysics ${ }^{\circledR}$. Variants of this simulation model have been used in previous work and published with details in [9] and [11]. The simulation model (or the environment) can be broken down as: (a) The Sensor; and (b) The Test Piece; these two components are described along with their parameters in Subsections IV-A and IV-B.

\section{A. Parameters of the PEC Sensor}

A cross section of the sensor placed above a test piece is shown in Fig. 3. The sensor is composed of an exciter coil and a detector coil as in Fig. 3 and as described in Subsection III-A. The lift-off created by the sensor casing is taken to be $4 \mathrm{~mm}$ as shown in Fig. 3 (i.e., the gap between the bottom of the detector coil and the top of the test piece). In practice, sensor casings are manufactured to be as thin as possible to avoid adding extra lift-off and are typically not thicker than about 5 $\mathrm{mm}$. Parameters of the sensor are given in Table I.

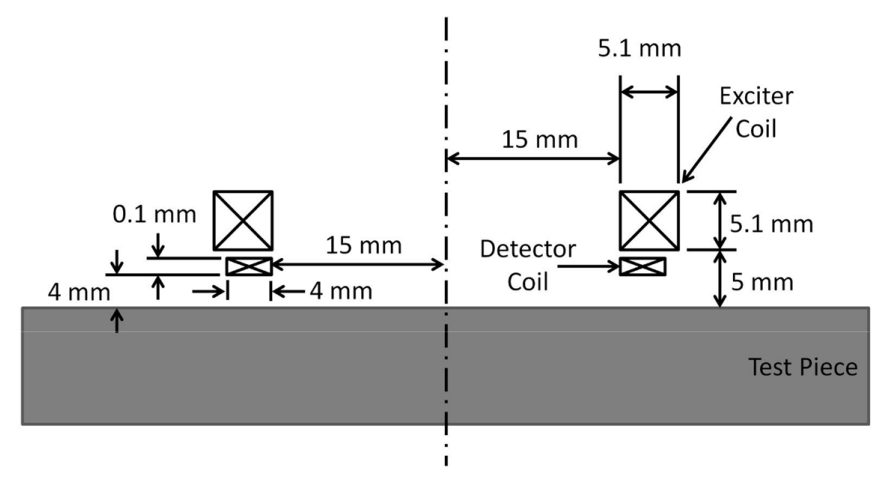

Fig. 3: Cross sectional view of the PEC sensor simulated for this paper.

TABLE I: Fixed Parameters of the PEC Sensor.

\begin{tabular}{c|c}
\hline Parameter & Value \\
\hline \hline Number of Exciter Coil Turns & 625 \\
\hline Exciter Coil Wire Gauge & AWG 32 \\
\hline Number of Detector Coil Turns & 50 \\
\hline Detector Coil Wire Gauge & AWG 40 \\
\hline Electrical Conductivity of Copper & $59.98 \mathrm{MS} / \mathrm{m}$ \\
\hline Magnetic Permeability of Copper & $0.4 \pi \mu \mathrm{H} / \mathrm{m}$ \\
\hline Excitation Current Pulse Amplitude & $100 \mathrm{~mA}$ \\
\hline
\end{tabular}

\section{B. Properties of the Test Piece}

Magnetic permeability $\left(\mu=\mu_{r} \mu_{0}\right)$ of the test piece was set to be such that the relative permeability $\mu_{r}=50$ to make the material ferromagnetic (somewhat similar to grey cast iron, 
a material on which PEC sensing is typically applied in the water industry [7]), and Electrical conductivity $\sigma=1.5 \mathrm{MS} / \mathrm{m}$ (again close to that of grey cast iron [9]). Nominal thickness was set to be $d_{0}=30 \mathrm{~mm}$ while to the radius of the test piece for 2D-Axisymmetric simulation was to set to be 150 $\mathrm{mm}$ to make the test piece considerably large with respect to the sensor whose outer radius is $20.1 \mathrm{~mm}$ (i.e., outer radius of the exciter coil domain).

For rust or graphitization layers, relative permeabilities were set such that $\mu_{t}=\mu_{b}=1$ and electrical conductivities were set such that $\sigma_{t}, \sigma_{b} \leq 0.1 \mathrm{MS} / \mathrm{m}$. It is shown in the results in Section $\mathrm{V}$ that $\sigma_{t}, \sigma_{b} \leq 0.1 \mathrm{MS} / \mathrm{m}$ do not make any significant impact on signals as $\sigma=1.5 \mathrm{MS} / \mathrm{m}$ and $\mu_{r}=50$ easily become dominant in influencing the signal. In return, this indicates that $\sigma_{t}, \sigma_{b} \leq 0.1 \mathrm{MS} / \mathrm{m}$ behaves the same way as $\sigma_{t}=\sigma_{b}=0$ (i.e., as air), making it easier to solve the problem by executing Step 2 of the solution proposed in Subsection III-C.

\section{RESUlts}

\section{A. Validation of the Simulation Model against Principles}

Prior to proceeding with demonstrating the solution, the simulation model was validated against principles. Suppose the case where a particular thickness $d$ is simulated while setting $l=0$ and $d_{0}=d$ and using the parameter values provided in Section IV. For this case, $\beta$ from the simulated signal can be obtained as described in Subsection III-B. On the other hand, $\beta$ in principle can be calculated as $\beta \approx \mu \sigma d^{2} / \pi^{2}$ [9] where $\mu$ and $\sigma$ take the values specified in Subsection IV-B. Thus obtained calculated and simulated $\beta$ values were compared in $\ln (\beta)$ form (another way of visualizing $\beta$ as done in [9]) to check the validity of the simulation model for $d$ values: $5 \mathrm{~mm}$, $10 \mathrm{~mm}, 15 \mathrm{~mm}, 20 \mathrm{~mm}$, and $25 \mathrm{~mm}$; all simulated by setting $l=0 \mathrm{~mm}$ and $d_{0}=d$. Table II shows the results which exhibit excellent agreement confirming the validity of the simulation model.

TABLE II: Comparison Between Simulated $\beta$ Values and Calculated $\beta$ Values.

\begin{tabular}{c|c|c}
\hline Thickness & $\ln (\beta)$ in Principle & $\ln (\beta)$ in Simulation \\
\hline \hline $5 \mathrm{~mm}$ & -8.34 & -8.34 \\
\hline $10 \mathrm{~mm}$ & -6.95 & -6.97 \\
\hline $15 \mathrm{~mm}$ & -6.14 & -6.13 \\
\hline $20 \mathrm{~mm}$ & -5.57 & -5.51 \\
\hline $25 \mathrm{~mm}$ & -5.12 & -5.11 \\
\hline
\end{tabular}

\section{B. Influence of $\sigma_{t}$ and $\sigma_{b}$ on PEC Signals}

Before attempting to solve the problem, it was important to identify the impact of $\sigma_{t}$ and $\sigma_{b}$ on a PEC signal. The simulation model was parametrised with $d=15 \mathrm{~mm}, l=15$ $\mathrm{mm}$, and $d_{0}=30 \mathrm{~mm}$, and simulations were run by varying $\sigma_{t}$ from 0 to $0.1 \mathrm{MS} / \mathrm{m}$. Usually such a range for electrical conductivity of rust or graphitization layer is realistic. Observed results presented in Fig. 4 suggest there is no significant impact on the signal (less that $1 \%$ variation in signal between 0 and $0.1 \mathrm{MS} / \mathrm{m}$ conductivity) and feature $\beta$ (variation less than $1 \%$ ) since $\mu_{t} \mu_{0}<<\mu$, as $\mu_{t}=1$ and $\sigma_{t}<\sigma$. It should be noted that the signal shift from the reference observed in this case is attributed to the effect of lift-off observed in Fig. 2 since the conductive ferromagnetic layer is $15 \mathrm{~mm}$ beneath the top surface of the test piece. Thereafter, simulations validate that the impact caused by $\sigma_{t}$ on the signal and its gradient is minimal.

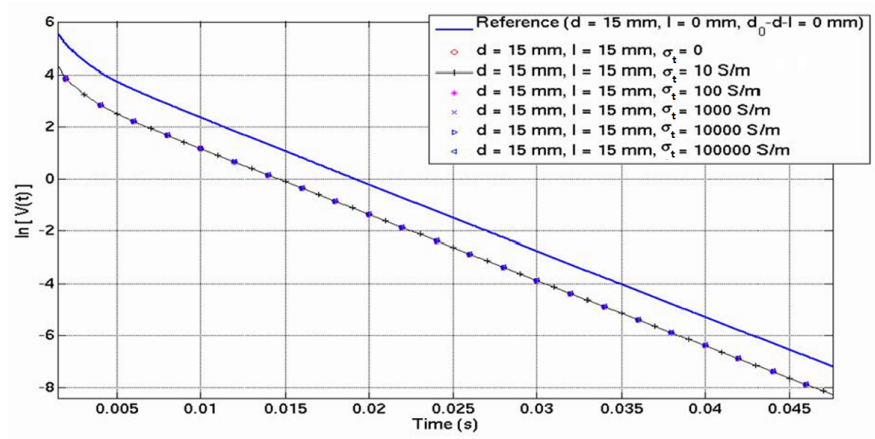

Fig. 4: Influence of $\sigma_{t}$ on the signal.

Similarly, to identify the influence of $\sigma_{b}$ on a PEC signal, the model was parametrized as $d=15 \mathrm{~mm}, d_{0}=30 \mathrm{~mm}$, and $l=0 \mathrm{~mm}$, and simulations were carried out with $\sigma_{b}$ in the range 0 to $0.1 \mathrm{MS} / \mathrm{m}$. Results in Fig. 5 suggest there is no significant impact (less than $1 \%$ variation) on the signal and $\beta$ since $\mu_{b} \mu_{0}<<\mu$ as $\mu_{b}=1$ and $\sigma_{b}<\sigma$. For the sake of completeness, simulations were run for the two cases by making $\sigma_{t}=\sigma_{b}=1 \mathrm{MS} / \mathrm{m}$ to verify the hypothesis that such high conductivity would invariably impact the signal, given the conductivity has reached the order of that of the conductive ferromagnetic material. A clear influence was observed as expected, but still in moderation since $\mu_{t}=\mu_{b}=1$ held. Significantly higher values of $\mu_{t}$ and $\mu_{b}$ should not usually exist in rust or graphitized layers.

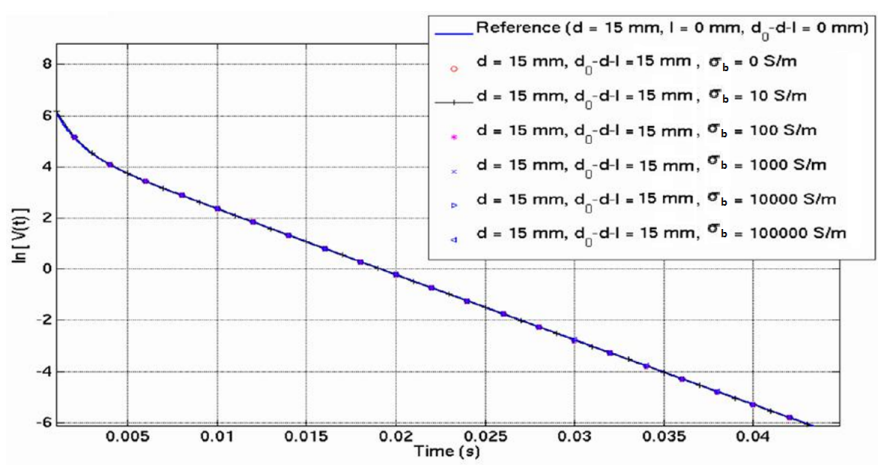

Fig. 5: Influence of $\sigma_{b}$ on the signal. 


\section{Estimating Wall Condition in $3 D$}

The learned function between $d$ and $\beta$ for the case $l=0$ and $d_{0}=d$ is shown in Fig. 6. The function was learned using GP as done in [9] and training was purely based on simulation. Since $\beta$ is independent of lift-off, and since rust or graphitized layers effectively create something similar to air lift-off as verified in Subsection V-B, the function in Fig. 6 could be used to estimate $d$ independent of $l$.

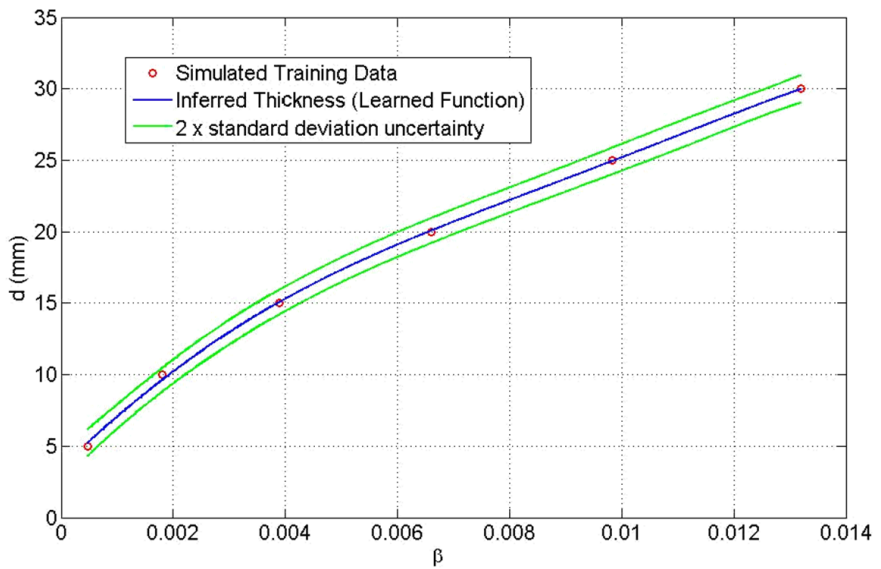

Fig. 6: GP-modelled function between thickness $(d)$ and signal feature $\beta$.

Different lift-off values (i.e., $l$ values considered by setting $\mu_{t}=\mu_{b}=1$ and $\sigma_{t}=\sigma_{b}=0 \mathrm{~S} / \mathrm{m}$ ) can be simulated for a known thickness $d$, and the threshold intersecting time $t_{t h}$ can be obtained for various lift-off values associated with $d$ as mentioned in Subsection III-C. A function between $l$ and thus obtained $t_{t h}$ for a known $d$ can then be modelled using GP in the same way done with the function between $d$ and $\beta$. A learned function between $l$ and $t_{t h}$ for $d=10 \mathrm{~mm}$ is shown in Fig. 7. Since the function between lift-off and threshold crossing time can thus be learned for any thickness, it is fundamentally possible to infer the thickness $(l)$ of the top layer when thickness $(d)$ of the ferromagnetic layer is known, enabling 3D profiling.

The 3D profiling capability was tested for several cases and Table III shows results for $d$ and $l$ estimates for a test case where $\sigma_{t}=1000 \mathrm{~S} / \mathrm{m}, \sigma_{b}=1500 \mathrm{~S} / \mathrm{m}, \mu_{t}=\mu_{b}=1$, and $d_{0}=30 \mathrm{~mm}$ were assumed to generate testing data through simulation. Since thickness and lift-off are estimated using GP, the estimates in Table III are provided with the two standard deviation uncertainty. The results in Table III thus demonstrate the capability of solving the 3D profiling problem.

\section{CONClusions}

This paper presented a framework based on solving the inverse PEC problem using the detector coil-based PEC sensor architecture, which fundamentally enables 3D representation of the condition of ferromagnetic wall-like structures. The framework involved dividing the test piece into three horizontal layers where the top and bottom layers represent rust/graphitization and the middle layer contains the healthy

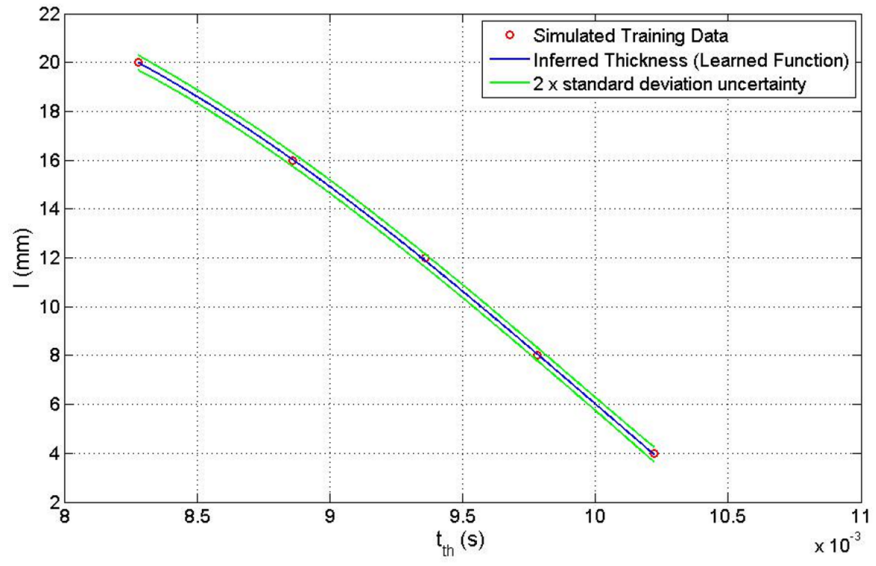

Fig. 7: GP-modelled function between $l$ and threshold crossing time $t_{t h}$ for $d=10 \mathrm{~mm}$.

TABLE III: 3D Profiling Capability Through Solving the Inverse Eddy Current Problem: Results.

\begin{tabular}{c|c|c|c}
\hline Actual $d$ & Estimated $d^{*}(\mathrm{~mm})$ & Actual $l$ & Estimated $l^{*}(\mathrm{~mm})$ \\
\hline \hline $7 \mathrm{~mm}$ & $6.66( \pm 0.856)$ & $17 \mathrm{~mm}$ & $16.997( \pm 0.583)$ \\
\hline $12 \mathrm{~mm}$ & $11.7( \pm 0.852)$ & $10 \mathrm{~mm}$ & $9.939( \pm 0.255)$ \\
\hline $17 \mathrm{~mm}$ & $17.1( \pm 0.863)$ & $8 \mathrm{~mm}$ & $7.901( \pm 0.188)$ \\
\hline $22 \mathrm{~mm}$ & $21.8( \pm 0.879)$ & $4 \mathrm{~mm}$ & $4.190( \pm 0.401)$ \\
\hline $27 \mathrm{~mm}$ & $26.1( \pm 0.952)$ & $2 \mathrm{~mm}$ & $1.99993( \pm 0.0248)$ \\
\hline
\end{tabular}

conductive ferromagnetic material. The rust or graphitized layers are usually non-magnetic, however, there is no guarantee they are non-conductive. Simulations suggested that the electrical conductivities of top and bottom layers did not noticeably influence PEC signals until they became very large. Since it is known that rust and graphitized layers cannot have high electrical conductivities, and moderate electrical conductivities do not significantly influence the signals, the paper demonstrated that it is reasonable to model them as layers having a relative permeability of 1 and a zero electrical conductivity (i.e., similar to the properties of air or free space). It was hence possible to estimate the thickness of the ferromagnetic material jointly with the thickness of the top layer as lift-off. Estimating the thickness of the top layer was done by means of learning a function between a threshold crossing time and lift-off. Since the function between liftoff and the threshold crossing time can be learned for any thickness, the paper showed that it is fundamentally possible to estimate the thickness of the top layer when thickness of the ferromagnetic layer has been estimated, hence making 3D profiling a possibility. The solution was demonstrated only in simulation, future work should involve practical implementations and experimental investigation.

\section{REFERENCES}

[1] J. García-Martín, J. Gómez-Gil, and E. Vázquez-Sánchez, "Nondestructive techniques based on eddy current testing," Sensors, vol. 11, 
no. 3, pp. 2525-2565, 2011.

[2] A. M. N. N. B. Ulapane, "Nondestructive evaluation of ferromagnetic critical water pipes using pulsed eddy current testing," $\mathrm{Ph}$.D. dissertation, 2016.

[3] C. Huang, W. Xinjun, X. Zhiyuan, and Y. Kang, "Pulsed eddy current signal processing method for signal denoising in ferromagnetic plate testing," NDT \& E International, vol. 43, no. 7, pp. 648-653, 2010.

[4] C. Huang, X. Wu, Z. Xu, and Y. Kang, "Ferromagnetic material pulsed eddy current testing signal modeling by equivalent multiple-coilcoupling approach," NDT \& E International, vol. 44, no. 2, pp. 163-168, 2011.

[5] X. Chen and Y. Lei, "Excitation current waveform for eddy current testing on the thickness of ferromagnetic plates," NDT \& E International, vol. 66, pp. 28-33, 2014.

[6] C. Huang and X. Wu, "An improved ferromagnetic material pulsed eddy current testing signal processing method based on numerical cumulative integration," NDT \& E International, vol. 69, pp. 35-39, 2015.

[7] J. V. Miro, J. Rajalingam, T. Vidal-Calleja, F. de Bruijn, R. Wood, D. Vitanage, N. Ulapane, B. Wijerathna, and D. Su, "A live test-bed for the advancement of condition assessment and failure prediction research on critical pipes," in Proceedings of the Leading-Edge Strategic Asset Management Conference (LESAM13), 2013.
[8] R. Petersen, R. Melchers et al., "Long-term corrosion of cast iron cement lined pipes," Centre for Infrastructure Performance and Reliability, vol. 23, pp. p1-10, 2012.

[9] N. Ulapane, A. Alempijevic, T. Vidal Calleja, and J. Valls Miro, "Pulsed eddy current sensing for critical pipe condition assessment," Sensors, vol. 17 , no. 10 , p. 2208,2017

[10] N. Ulapane, A. Alempijevic, T. Vidal-Calleja, J. V. Miro, J. Rudd, and M. Roubal, "Gaussian process for interpreting pulsed eddy current signals for ferromagnetic pipe profiling," in Industrial Electronics and Applications (ICIEA), 2014 IEEE 9th Conference on. IEEE, 2014, pp. 1762-1767.

[11] N. Ulapane, L. Nguyen, J. Valls Miro, A. Alempijevic, and G. Dissanayake, "Designing a pulsed eddy current sensing set-up for cast iron thickness assessment," in 12th IEEE Conference on Industrial Electronics and Applications, 2017.

[12] L. Nguyen, N. Ulapane, J. Valls Miro, G. Dissanayake, and F. Munoz, "Improved signal interpretation for cast iron thickness assessment based on pulsed eddy current sensing," in 12th IEEE Conference on Industrial Electronics and Applications, 2017.

[13] C. E. Rasmussen, "Gaussian processes in machine learning," in Advanced lectures on machine learning. Springer, 2004, pp. 63-71. 